\title{
Video Article \\ Flat-floored Air-lifted Platform: A New Method for Combining Behavior with Microscopy or Electrophysiology on Awake Freely Moving Rodents
}

\author{
Mikhail Kislin ${ }^{1}$, Ekaterina Mugantseva ${ }^{1}$, Dmitry Molotkov ${ }^{1}$, Natalia Kulesskaya ${ }^{1}$, Stanislav Khirug ${ }^{1}$, llya Kirilkin ${ }^{2}$, Evgeny Pryazhnikov ${ }^{1,2}$, \\ Julia Kolikova ${ }^{2}$, Dmytro Toptunov ${ }^{2}$, Mikhail Yuryev ${ }^{1}$, Rashid Giniatullin ${ }^{3}$, Vootele Voikar ${ }^{4}$, Claudio Rivera ${ }^{1}$, Heikki Rauvala ${ }^{1}$, Leonard Khiroug ${ }^{1}$ \\ ${ }^{1}$ Neuroscience Center, University of Helsinki \\ ${ }^{2}$ Neurotar LTD \\ ${ }^{3}$ A. I. Virtanen Institute for Molecular Sciences, University of Eastern Finland \\ ${ }^{4}$ Laboratory Animal Center, University of Helsinki
}

Correspondence to: Leonard Khiroug at leonard.khirug@helsinki.fi

URL: https://www.jove.com/video/51869

DOI: doi:10.3791/51869

Keywords: Issue 88, awake, in vivo two-photon microscopy, blood vessels, dendrites, dendritic spines, $\mathrm{Ca}^{2+}$ imaging, intrinsic optical imaging, patch-clamp

\section{Date Published: 6/29/2014}

Citation: Kislin, M., Mugantseva, E., Molotkov, D., Kulesskaya, N., Khirug, S., Kirilkin, I., Pryazhnikov, E., Kolikova, J., Toptunov, D., Yuryev, M., Giniatullin, R., Voikar, V., Rivera, C., Rauvala, H., Khiroug, L. Flat-floored Air-lifted Platform: A New Method for Combining Behavior with Microscopy or Electrophysiology on Awake Freely Moving Rodents. J. Vis. Exp. (88), e51869, doi:10.3791/51869 (2014).

\section{Abstract}

It is widely acknowledged that the use of general anesthetics can undermine the relevance of electrophysiological or microscopical data obtained from a living animal's brain. Moreover, the lengthy recovery from anesthesia limits the frequency of repeated recording/imaging episodes in longitudinal studies. Hence, new methods that would allow stable recordings from non-anesthetized behaving mice are expected to advance the fields of cellular and cognitive neurosciences. Existing solutions range from mere physical restraint to more sophisticated approaches, such as linear and spherical treadmills used in combination with computer-generated virtual reality. Here, a novel method is described where a headfixed mouse can move around an air-lifted mobile homecage and explore its environment under stress-free conditions. This method allows researchers to perform behavioral tests (e.g., learning, habituation or novel object recognition) simultaneously with two-photon microscopic imaging and/or patch-clamp recordings, all combined in a single experiment. This video-article describes the use of the awake animal head fixation device (mobile homecage), demonstrates the procedures of animal habituation, and exemplifies a number of possible applications of the method.

\section{Video Link}

The video component of this article can be found at https://www.jove.com/video/51869/

\section{Introduction}

An exciting recent trend in the Neurosciences is to develop experimental approaches for molecular and cellular probing of neuronal networks in the brain of awake, behaving rodents. Such approaches hold promise to shed new light on neurophysiological processes that underlie motor function, sensorimotor integration, perception, learning, memory, as well as injury progression, neurodegeneration and genetic diseases. Furthermore, recording from awake animal's brain holds promise in development of novel therapeutic agents and treatments.

There is a growing awareness that anesthesia, which has been commonly used in neurophysiological experiments, can affect the basic mechanisms of brain function, potentially leading to erroneous interpretation of experimental findings. Thus, the widely used anesthetic ketamine rapidly increases formation of new dendritic spines and enhances synaptic function ${ }^{1}$; another commonly used anesthetic isoflurane at surgical anesthesia levels completely suppresses spontaneous cortical activity in new-born rats and blocks spindle-burst oscillations in adult animals ${ }^{2}$. At present, only a limited number of approaches enable experiments in non-anesthetized mice by means of two-photon microscopic imaging or patch-clamp recordings. These approaches can be divided into freely moving and head-fixed preparations.

The unique attractiveness of a freely moving animal preparation is that it allows assessment of natural behavior, including whole body movements during navigation. One way to image inside the brain of a freely moving rodent is to attach a miniaturized head-mounted microscope or fiberscope ${ }^{3-5}$. However, miniaturized devices tend to have limited optical performance in comparison to objective-based two-photon microscopy, and cannot be easily combined with whole cell patch-clamp recordings 6 .

The existing solutions for head-fixing an awake rodent have relied primarily either on physical restraint ${ }^{7,8}$ or on training the animal to exhibit voluntary head restraint ${ }^{9}$. Another popular approach is to allow the animal's limbs to move by placing it on, e.g., a spherical treadmill ${ }^{10}$; this approach is often combined with computer-generated virtual reality. Electrophysiological experiments on head-fixed mice have mostly used extracellular recordings and were used to study central regulation of cardiovascular function ${ }^{11}$, effects of anesthesia on neuronal activity ${ }^{12}$, the auditory response in the brainstem ${ }^{13}$ and information processing ${ }^{14}$. The pioneering intracellular/whole-cell recordings in awake behaving 
animals were performed in the 2000 s and have focused on neural activity related to perception and motion ${ }^{15-20}$. Around the same time, the first microscopic imaging studies on awake mice were published, where two-photon microscopy was used in the sensory cortex of physically restrained rats ${ }^{7}$ and on mice running on a spherical treadmill ${ }^{21}$

Subsequent in vivo microscopy and electrophysiology studies demonstrated that a head fixation preparation can be successfully combined with behavioral paradigms based on forelimb movements, odor recognition, whisking, and licking ${ }^{8,22-25}$. Mice placed on the spherical treadmill can be trained to navigate the virtual visual environment generated by a computer ${ }^{10,26}$. Intracellular/extracellular recordings demonstrated that, in a head-fixed animal navigating such virtual environment, activation of hippocampal place cells can be detected ${ }^{27}$. In a virtual visual environment, mice demonstrate normal movement-related theta rhythm in the local field potential and theta-phase precession during active movement ${ }^{27}$. Recently, the spatial and temporal activity patterns of neuronal populations were recorded optically in mice during working memory decision tasks in a virtual environment ${ }^{28}$.

Despite having enabled breakthrough research, the spherical treadmill design has several inherent limitations. First, the animal is required to move on an unlimited surface of a rotating air-lifted ball, which poses no tangible obstacles such as walls or barriers. This limitation is only in part compensated by the computer-generated "virtual reality", because visual input is arguably less effective on mice and rats as compared to the tactile sensory input (e.g., whisker-touch or lick), which these species naturally rely on. Second, the considerable curvature of the ball surface may be uncomfortable for the laboratory mice used to walking on a flat floor in their cages. Finally, the sheer diameter of the ball (at least 200 $\mathrm{mm}$ for mice and $300 \mathrm{~mm}$ for rats) renders the vertical size of the spherical treadmill device relatively large. This makes it difficult to combine spherical treadmill with the majority of commercially available microscopy setups, and often requires building a new setup around the treadmill by means of custom-made microscope frames.

Here, a novel method is described where a head-fixed mouse can move around an air-lifted mobile homecage that features a flat floor and tangible walls, and explore the physical environment under stress-free conditions. This article demonstrates the procedures of mouse training and head fixation, and provides representative examples where two-photon microscopy, intrinsic optical imaging and patch-clamp recordings are performed in the brain of awake behaving mice.

\section{Protocol}

All the procedures presented here were performed according to local guidance for animal care (The Finnish Act on Animal Experimentation (62/2006)). The animal license (ESAVI/2857/04.10.03/2012) was obtained from local authority (ELÄINKOELAUTAKUNTA-ELLA). Adult mice (age 1-3 months, weight 20-40 g) were kept in group-housing cages in the certified animal facility of the University of Helsinki and provided with food and water ad libitum.

\section{Cranial Window Implantation}

Cranial window is implanted according to published protocols ${ }^{29-31}$ with minor modifications, as briefly described below:

1. Sterilize the instruments before starting the cranial window implantation. Maintain sterile conditions during surgery to minimize the risk of postoperative complications.

2. Administer an analgesic (Ketoprofen, $2.5 \mathrm{mg} / \mathrm{kg}$ ) $30 \mathrm{~min}$ before the surgery and $24 \mathrm{hr}$ post-surgery. Anesthetize the mouse using a mixture of ketamine $(80 \mathrm{mg} / \mathrm{kg})$ and xylazine $(10 \mathrm{mg} / \mathrm{kg})$ injected intraperitoneally. Regularly monitor the depth of anesthesia by paw pinching. Use a heating pad to maintain the animal's body temperature at $37.0^{\circ} \mathrm{C}$. To reduce the surgery-induced inflammation and cerebral edema, administer dexamethasone $(2 \mathrm{mg} / \mathrm{kg})$ by sub-cutaneous injection.

3. Apply eye lubricant to protect the eyes from getting dry. Shave the mouse's head and clean the shaved area. Cut the skin using surgical scissors and forceps along the line from the scruff to the forehead. Remove any connective tissue attached to the skull.

4. Slowly and carefully drill a small well on the left frontal bone using a high speed surgical drill. Screw a mini bolt (see Materials) into the drilled well. Perform no more than one-and-a-half full turns of the screw.

NOTE: Avoid those areas that are located directly above the superficial cortical vessels. Disruption of these vessels can lead to massive bleeding.

5. To perform craniotomy, drill a circular window (3-3.5 mm in diameter) in the right parietal bone. Apply a drop of the cortex buffer (125 mM $\mathrm{NaCl}, 5 \mathrm{mM} \mathrm{KCl}, 10 \mathrm{mM}$ glucose, $10 \mathrm{mM} \mathrm{HEPES}, 2 \mathrm{mM} \mathrm{CaCl}_{2}$, and $2 \mathrm{mM} \mathrm{MgSO}_{4}$ in distilled $\mathrm{H}_{2} \mathrm{O}$ supplemented with penicillin 100 units/ml and streptomycin $100 \mu \mathrm{g} / \mathrm{ml}$ ) and carefully remove the part of the bone located inside the circular window. NOTE: To express a fluorescent protein in a specific subpopulation of cells, perform intracranial injection of adeno-associated viral (AAV) vector or other viral vectors at this point in the procedure.

6. Position a round glass coverslip (\#1.5 thickness code) on the cranial window. Attach the coverslip to the skull with polyacrylic glue. Place a metal holder on top of the coverslip and fix it with the mixture of dental cement and polyacrylic glue.

NOTE: The procedure described above is optimized for cranial windows used in optical imaging experiments. To prepare an "inverted" cranial window suitable for electrophysiological experiments, use a different procedure. First, glue the metal holder to the skull. Drill a circular window within the holder's opening as exemplified in the video. Alternatively, make a smaller craniotomy (less than $0.5 \mathrm{~mm}$ in diameter) over the region of interest to prevent or minimize the brain movement ${ }^{32}$. Refresh the cortical buffer or place a drop of silicone adhesive on the cranial window, then close it with a round glass coverslip.

7. After completing the operation, place the animal in a warmed cage with easily accessible food and water. Return the animal to the grouphousing cage only after it recovers fully from anesthesia. Re-administer analgesic upon detecting any signs of pain, including reluctance to move, eat or drink, weight loss, salivation, piloerection or abnormal respiratory sounds.

\section{Animal Handling}

1. Take the mouse from its cage and simply hold it for 5-10 min. Be calm while handling the animal, avoid making jerky movements and noises. 
2. After handling, return the mouse to its cage.

3. Repeat the handling procedure $2-3 x$ with unequal intervals between handling episodes, in order to make the mouse comfortable with the experimentalist.

4. Take a small soft rag and wrap the animal $2-3 x$ with unequal intervals.

5. Animal should remain calm and get accustomed to being wrapped. If the mouse is excited and nervous, repeat the handling and wrapping procedures.

\section{Animal Training}

1. Start training of the animal in the mobile homecage the next day after the habituation to handling and wrapping is completed.

2. Record the animal's weigh daily before and during training NOTE: If, during the training, the animal loses over $10 \%$ of its weight, it should be excluded from the experiments.

3. Adjust the vertical position of the head fixation arm of the mobile homecage device in order to match the size of the trained animal. Connect the air inlet of the device to the standard laboratory pressurized air outlet (typically, either a gas tank or an air-pump that provides a sufficiently high pressure and rate of the air flow, i.e. approximately 5 bar and $300 \mathrm{l} / \mathrm{min}$ ).

4. Wrap the animal in a rag.

5. Insert the metal holder, which is attached to the animal's head, into the head fixation arm and firmly fix it by tightening the screws. Turn on the air flow and make sure that the air flow is optimal for free flotation of the air-lifted homecage. Release the animal into the mobile homecage by removing the rag.

6. To habituate the animal to noise, provide a constant exposure to ambient sounds (using, for example, the radio or recorded music and speech) during all training sessions as well as during the experiments.

7. During the first training session, keep the room light on for the first hour, then turn off the light until the end of the training session.

8. After $2 \mathrm{hr}$ of training in the mobile homecage, release the animal from head fixation and return it to its cage with a supply of water and food. Leave it for at least $2 \mathrm{hr}$ under resting conditions.

9. Clean the mobile homecage after each training session using a $70 \%$ ethanol solution and rinse it with tap water. Soak-up the water with disposable paper towel and dry the mobile homecage before the next use.

10. Perform consecutive training sessions for $2 \mathrm{hr}$, with the room light turned off while the animal is being trained.

11. Perform the training session twice daily.

12. After 8 to 12 training sessions, use the animal in an experimental session for up to $2 \mathrm{hr}$ at a time.

NOTE: During prolonged training sessions that last more than 1-2 hr, consider providing the mouse with drinking water, which can be delivered either manually or using a pipette holder attached to the mobile homecage frame. Alternatively, water can be supplied for ad libitum use of the animal by placing viscous drops of hydro-gel directly on the walls of the mobile homecage.

NOTE: Remember to weigh the animals every day before training to rule out any chronic stress effects. Exclude an animal from the experiment if, at any time point, it demonstrates stress reactions such as freezing, vocalization, or stress-induced diarrhea.

\section{Applications}

1. Two-photon Imaging in Awake Mouse Moving Around the Mobile Homecage

1. Assemble the mobile homecage. Check positions of the bridge and head fixation arm.

2. Wrap the trained animal in a rag. Place the animal in the mobile homecage. Clamp the metal holder in the head fixation arm. Remove the rag.

3. Clean the implanted cover glass from dust using a $70 \%$ ethanol solution and allow it to dry.

4. Place a drop of the immersion fluid on the cover class. Preferably, use a viscous solution, because water will evaporate quickly.

5. Place the mobile homecage device with the trained animal under the microscope (unless you have a second, identical device, which is stationary in the microscopy setup).

6. Perform the imaging using either a custom made or a commercially available laser-scanning microscope imaging system equipped with a femtosecond pulsed infrared laser.

7. Find the region of interest using the wide-field mode of the fluorescence microscope. Use long pass filters to assess brain vasculature and select an appropriate target area after examining the pattern of blood vessels.

8. To image cortical vasculature, inject a $1 \%$ solution of the $70,000 \mathrm{MW}$ Texas Red-conjugated dextran (or its analogue), either into the tail vein while the animal is immobilized in the rag, or retro-orbitally while the animal is positioned in the mobile homecage. Tune the two-photon laser to $860 \mathrm{~nm}$ and use the band pass filter $(590-650 \mathrm{~nm})$ to collect the emitted light. Use the 515-560 nm emission filter to assess the fine details of neuronal morphology or neuronal activity utilizing, for example, transgenic mice that express $\mathrm{YFP}_{\mathrm{F}}$ or the Ca ${ }^{2+}$ sensitive fluorescent protein GCaMP3 in a subpopulation of neurons under the Thy1 promoter.

9. Use an appropriate software for image acquisition.

10. After imaging, release the animal from the head fixation arm by loosening the screws. Return the animal to its cage and allow it to rest for at least $2 \mathrm{hr}$ before starting the next imaging session.

11. Store the coordinates of every region of interest (ROI) for subsequent reimaging. Image the same ROls over time, and adjust coordinates each time to maximize the image overlap.

12. Analyze the images and make three-dimensional reconstructions using an appropriate software (e.g., ImageJ, etc.).

2. Intrinsic Optical Imaging in Awake Mouse Moving Around the Mobile Homecage

1. Assemble the mobile homecage under the image acquisition camera of an intrinsic optical imaging setup.

2. Wrap the trained animal in a rag. Place the animal in the mobile homecage. Clamp the metal holder into the head fixation arm.

3. Clean the implanted cover glass from dust using a $70 \%$ ethanol solution and allow it to dry.

4. Place a drop of glycerol on the implanted glass and cover it with an $8 \mathrm{~mm}$ round cover slip.

5. Place a manipulator with the air-blow tube opposite to the contralateral vibrissa. 
6. Adjust the position of the high-speed camera and focus it on the cortical vasculature.

7. Use green light (filter 546BP30) without a camera filter to acquire the vessels map.

8. Focus deeper into the cortex, approximately 400 micrometers below the cortical surface.

9. To image the blood oxygenation level-dependent (BOLD) optical signal, place the 590LP filter in front of the camera and illuminate the cortex with red light (filter 630BP30).

10. Adjust the illumination of the cortical surface so that it is evenly distributed through the region of interest, avoiding overexposure. Adjust the illumination intensity so that the region of interest falls within the $70-90 \%$ segment of the camera's dynamic range.

11. Use LongDaq image acquisition software to collect images from the camera.

12. Use image acquisition frequency of 1 to $10 \mathrm{~Hz}$ (i.e., between 1 and 10 frames per second) for experiments with low frequency stimulation $(0.05 \mathrm{~Hz})$.

13. Switch off the room light to prevent any interference with the intrinsic optical signal.

14. Allow at least $30 \mathrm{~min}$ for the mouse to adapt to the mobile homecage.

15. Image the baseline activity during a 6-min episode without stimulation.

16. To record evoked cortical activity, stimulate vibrissa in a $10 \mathrm{sec}$ ON/10 sec OFF mode ( $0.05 \mathrm{~Hz}$ stimulation) with a high frequency (25 $\mathrm{Hz}$ ) train of air puffs for the total period of $6 \mathrm{~min}$.

17. After image acquisition, release the mouse from the head fixation arm and return it to its cage.

18. Do not use additional data filtering for frequency, because the amplitudes of stimulation responses tend to be relatively high in awake animals, resulting in excellent signal to noise ratio.

19. Convert the obtained sets of images to *.tif stack files and analyze them further using, e.g., the open source FIJI software (ImageJ). Subtract the baseline spontaneous activity from the frames obtained during vibrissa stimulation using the Image Calculator tool. Alternatively, filter data in the frequency domain using appropriate software.

3. Patch-clamp Recordings in Awake Mouse Moving Around the Mobile Homecage

1. Assemble the mobile homecage.

2. Wrap the trained animal in a rag. Administer trimethoprime $(5 \mathrm{mg} / \mathrm{kg})$ and sulfadoxine $(25 \mathrm{mg} / \mathrm{kg})$ to prevent bacterial infection. Place the animal in the mobile homecage. Clamp the metal holder into the head fixation arm.

3. Clean and sterilize the implanted dental cement "cap" and cover glass using a $70 \%$ ethanol solution or $0.5 \%$ chlorhexidine digluconate and allow it to dry.

4. Slowly and carefully remove the cover glass from the metal holder.

5. Refresh the cortical buffer supplemented with penicillin, streptomycin and clean the cranial window from debris with a sterile hemostatic tampon.

6. Place the ground electrode into the cortical buffer.

7. Place the electrophysiology headstage in a micromanipulator.

8. Fabricate pipettes from borosilicate glass, aiming at tip resistance ranging from 6.5 to $8.5 \mathrm{M} \Omega$. Fill the patch pipette with an intracellular solution. The composition of the patch pipette solution is the following (in $\mathrm{mM}$ ): $8 \mathrm{KCl}, 111 \mathrm{~K}$-gluconate, $0.5 \mathrm{CaCl}_{2}, 2 \mathrm{NaOH}, 10$ glucose, $10 \mathrm{HEPES}, 2 \mathrm{Mg}$-ATP, and $5 \mathrm{BAPTA}$, pH was adjusted to 7.2 with $\mathrm{KOH}$. The membrane potential values must be corrected for a calculated liquid junction potential of $-12 \mathrm{mV}^{33}$.

9. Target the region of interest using stereotaxic coordinates, and quickly move the electrode into the brain while maintaining strong positive pressure on the pipette tip. After the dura mater penetration and the pipette positioning, measure the tip resistance and discard the electrodes that show an increase in resistance of more than $10-15 \%$, in order to improve the success rate of the subsequent steps.

10. Reduce the positive pressure by half to avoid swelling of the surrounding brain tissue. Further steps are similar to the standard "blind patch" protocol. To find a neuron to record from, lower the tip into the brain in a stepwise fashion until a neuron is detected in a close proximity of the pipette tip, as indicated by a characteristic temporal sequence of electrode impedance changes. The key indicator of the presence of a neuron is a monotonic increase in electrode resistance across several consecutive forward steps of the pipette (typically, a $20 \%$ increase in the pipette resistance across three $2-\mu \mathrm{m}$ steps).

11. To form a gigaseal contact with the targeted neuron, apply a negative pressure and hyperpolarization of the pipette.

12. Apply a brief pulse of a larger negative pressure to the cell in order to establish the whole-cell configuration. Retract the electrode by 2-3 $\mu \mathrm{m}$ to keep a good seal.

13. Record spontaneous or evoked activity during a desired period of time, up to $20-40 \mathrm{~min}$.

14. After recording, remove the pipette from the brain.

15. Refresh the cortical buffer or place a drop of silicone adhesive on the cranial window, then glue a round glass coverslip on top of the metal holder.

16. Release the animal from the head fixation arm by loosening the screws. Return the animal to its cage for at least one day before the next recording.

17. Analyze data with, e.g., the FitMaster software.

4. Habituation-dishabituation Olfactory Test in Awake Mouse Moving Around the Mobile Homecage

1. Attach a clean cotton piece $(2 \times 2 \mathrm{~cm})$ dipped in tap water to the inner side of the wall of the mobile homecage using a two-sided tape. Divide the mobile homecage wall into four zones by placing color markers on the outer side of the wall so that the piece of cotton is located in the middle of the "target zone". Fix the animal in the headholder arm facing the wall segment opposite to the "target" zone and allow it to adapt to the mobile homecage for $30 \mathrm{~min}$.

2. Take another piece of clean cotton and wet it with a few drops of the $1 \%$ vanilla extract. Replace the clean cotton on the mobile homecage wall with the one carrying the vanilla smell. Present the smell for $5 \mathrm{~min}$. Track the movements of the mobile homecage during the smell presentation period. Estimate the level of interest to the smell by measuring the cumulative time that the animal spends facing the "target" zone in relation to the total time of mobile homecage movement.

3. Repeat the 5-min application session three times using the smell of vanilla, with a 5 min inter-session interval. Use a fresh piece of "vanilla" cotton in every session.

4. Present the animal with a socially significant smell during the last, fifth session. Wet a clean piece of cotton with few drops of urine (obtained on the preceding day from an animal of the opposite gender) and place it in the middle of the target zone for 5 min. 
5. Novel smell recognition in awake mouse moving around the mobile homecage

1. Divide the wall into four zones by placing color markers on the outer side of the mobile homecage wall. Attach two clean cotton pieces $(2 \times 2 \mathrm{~cm})$ dipped in tap water to the inner side of the wall in the middle of the zones opposing each other (designated as target zone 1 and target zone 2). Fix the animal in the headholder arm facing a wall segment outside of the target zones and allow it to adapt to the mobile homecage for $30 \mathrm{~min}$.

2. Replace both cotton pieces with the fresh ones: place a cotton piece wet with the $1 \%$ vanilla extract to target zone 1 and another one wet with tap water to target zone 2. Record the video of the mobile homecage movements for 10 min during the smell presentation session. Calculate the smell attending as the percentage of the time that the animal spends facing target zone 1 relative the cumulative time spent facing zones 1 and 2.

3. After a $10 \mathrm{~min}$ interval, place another pair of applicators on the homecage wall for the subsequent 10 min period. Place the "vanilla" cotton in target zone 1 and cotton applicator wet with $1 \%$ banana extract in target zone 2 . Make a video recording of the mobile homecage movements. Calculate the preference to novel smell as the percentage of time that the animal spends facing the wall segment with the novel smell (target zone 2) relative to the cumulative time facing zones 1 and 2.

\section{Representative Results}

The method presented here is intended for microscopic imaging or single-cell electrophysiological recordings in awake, head-fixed but otherwise freely-moving and behaving mice. The animal can move in two dimensions in a real (as opposed to virtual), tangible and familiar environment, while the animals' skull is fixed firmly to the head fixation arm. Habituating the mice to the air-lifted mobile homecage consists of 4-6 days of twice-daily 2-hr training sessions (Figure 1). The trained animals can then be used in the experiments immediately. A typical study includes a number of imaging sessions or patch-clamp recording sessions that are spaced at intervals ranging from few hours to several days or weeks. Importantly, both optical and electrophysiological recordings can be performed simultaneously with cognitive or behavioral stimuli and readouts, within a single experiment.

To evaluate the mechanical stability of mouse's head fixation in the mobile homecage, the image sequences of cortical vessels labeled with fluorescent-conjugated dextran and of cortical dendrites expressing YFP were collected while the experimental animals were navigating the mobile homecage (Figure 2). The maximal displacements of the brain during animal's locomotion did not typically exceed 1-1.5 micrometers. These displacements occurred in the horizontal directions and very rarely resulted in a detectable shift of the imaging plane, rendering unnecessary any correction of motion artifacts. Stable head fixation in mobile homecage allows quantification of individual dendritic spines in awake animals with the same reliability as in anesthetized mice. Dendritic spine density, morphology and turnover can be monitored during longitudinal studies with multiple imaging sessions performed at intervals ranging from a few hours to several days or weeks.

The usability of the mobile homecage for functional optical imaging was tested in somatosensory cortex of awake mice using two approaches: i) two-photon microscopy on the Thy1-GCaMP3 transgenic mice and ii) intrinsic optical signal imaging in wild-type mice. Ca ${ }^{2+}$ imaging was performed in layer $2 / 3$, which contains cell bodies of many fluorescently labeled neurons, as well as their dendrites and axons (Figure 3 ). The plots of fluorescence-over-time from selected regions of interest (ROIs) are shown in Figure 3, demonstrating spontaneous neuronal activity (measured as transient increases in GCaMP3 fluorescence) during the mouse's active navigation in the mobile homecage. Optical imaging based on intrinsic signals allows mapping the spatial distribution of functional domains. Figure 4 illustrates wave-like changes in the blood oxygenation level (reflecting regional neuronal activation) that propagated along somatosensory cortex in response to vibrissa stimulation at the frequency of $0.05 \mathrm{~Hz}$.

To test feasibility of patch-clamp recordings with mobile homecage, we used 2-3 months-old C57BI/6J mice. Layer 2/3 neurons in somatosensory cortex were recorded from in whole cell configuration using current clamp mode. Patch-clamp recording in the brain of awake mice head-fixed on mobile homecage was essentially similar to blind patch-clamping in brain slices. Approximately $50 \%$ of attempts resulted in successful gigaseal formation, of which more than $70 \%$ yielded stable whole-cell configuration recording. No events of losing gigaseal contact due to mechanical displacement of cells were observed. Figure 5 illustrates a 60 -sec fragment of a representative 10-min long current-clamp recording correlated with episodes of mouse's active (running) and passive (resting) states. 
A
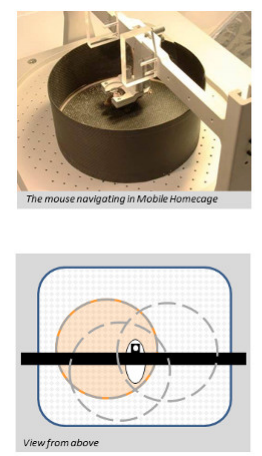
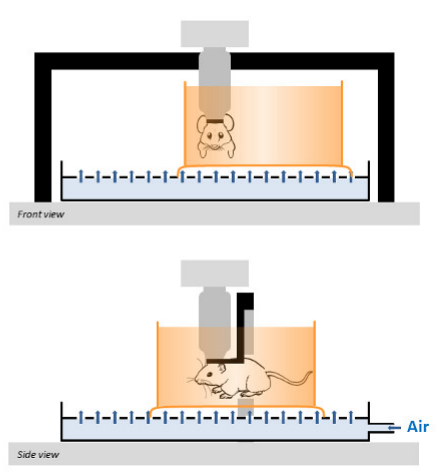

B

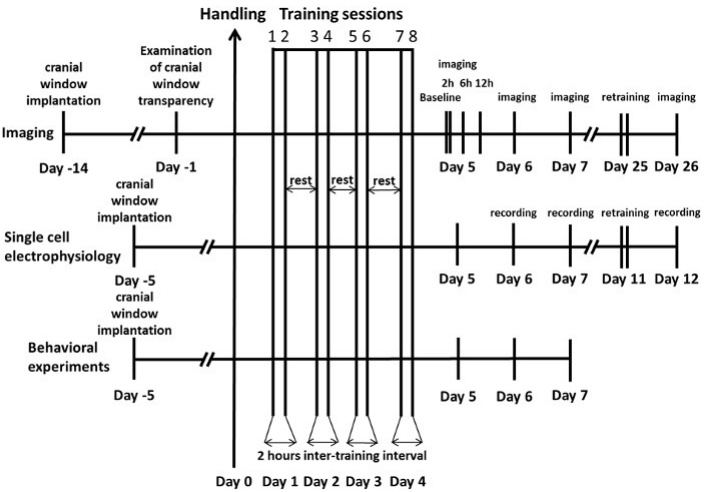

Figure 1. The method of head fixation of awake mice in the mobile homecage. A) Overview of the air-lifted mobile homecage design and illustrations of the general concept. B) Diagram of a typical experimental timeline. The study begins with implantation of the cranial window two weeks prior to habituating the mouse to handling and wrapping, which is followed by eight twice-daily training sessions. The typical study includes a number of imaging sessions or patch clamp recording sessions that are spaced at intervals ranging from few hours to several days or weeks. Both optical and electrophysiological measurements can be done in parallel with cognitive or behavioral stimuli and readouts within a single experiment. Please click here to view a larger version of this figure.
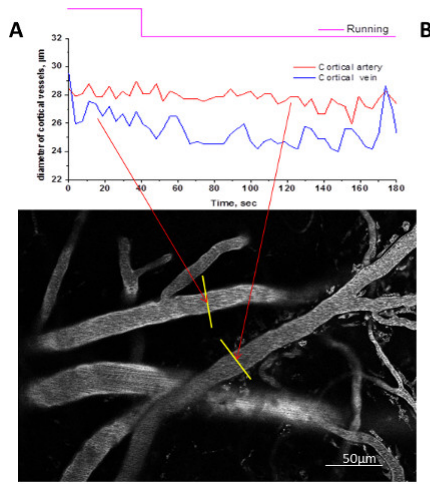

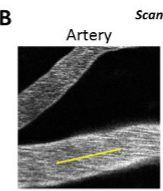

Velocity $0.97 \mathrm{~mm} / \mathrm{s}$

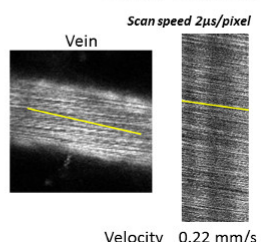

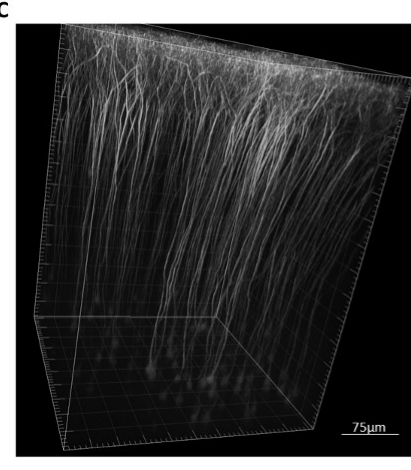

D
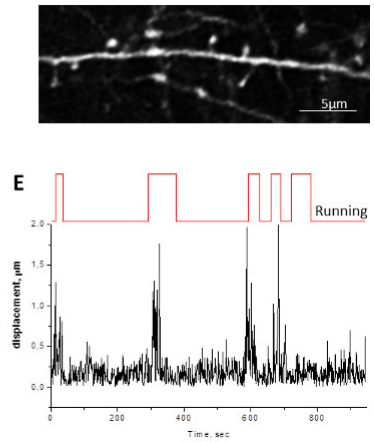

Figure 2. Example of two-photon microscopic imaging on awake mice moving around the mobile homecage. A,B) Cortical vasculature, labelled with the $70 \mathrm{kDa}$ Texas Red-conjugated dextran. The diameter of individual vessel segments is measured by plotting over time the profile of the lines drawn across the vessel lumen during periods of mouse's resting and running (A). The rate of blood flow in arteries and veins is measured by line-scanning along the lines drawn parallel to the vessel wall (B). C,D) Fine details of neuronal morphology visualized in the brain of transgenic mice that express YFP in subpopulation of neurons under the Thy1 promoter. Three-dimensional reconstruction of pyramidal neurons in the mouse's somatosensory cortex (C). The images of a dendritic branch acquired in an awake, behaving mouse are sufficiently stable for quantification of individual dendritic spine morphology (D). E) Quantification of brain motion caused by mouse movements. Larger amplitude displacements correlate with periods of the mouse's running. Please click here to view a larger version of this figure. 
Spontaneous $\mathrm{Ca}^{2+}$ - spikes in awake Thy1-GCamp3 mouse navigating around the mobile homecage
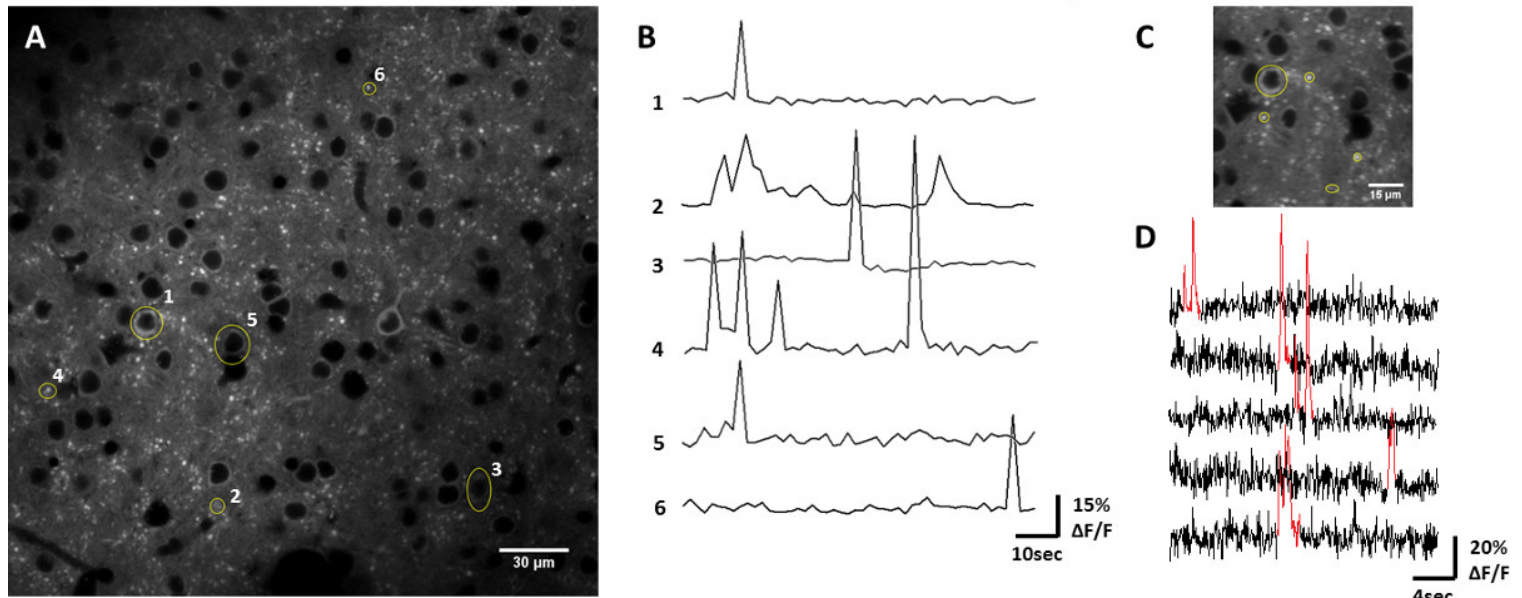

Figure 3. Example of neuronal population activity in awake Thy1-GCaMP3 mouse moving around the mobile homecage. A) Twophoton image of cortical layer II/III neurons. ROls, for example neuronal cell bodies, dendrites and axons are shown in yellow. B) $\Delta \mathrm{F} / \mathrm{F}$ traces of the GCaMP3 fluorescence from ROls shown in A (time series recorded at $1.5 \mathrm{sec} / \mathrm{frame}$ ). C) Zoomed-in region imaged at $65 \mathrm{msec} / \mathrm{frame}$. D) Fluorescence from yellow ROIs in C plotted over time, shows the transient increases (red) in the GCaMP3 fluorescence that correspond to the action potential-induced $\mathrm{Ca}^{2+}$ influx episodes. Please click here to view a larger version of this figure.
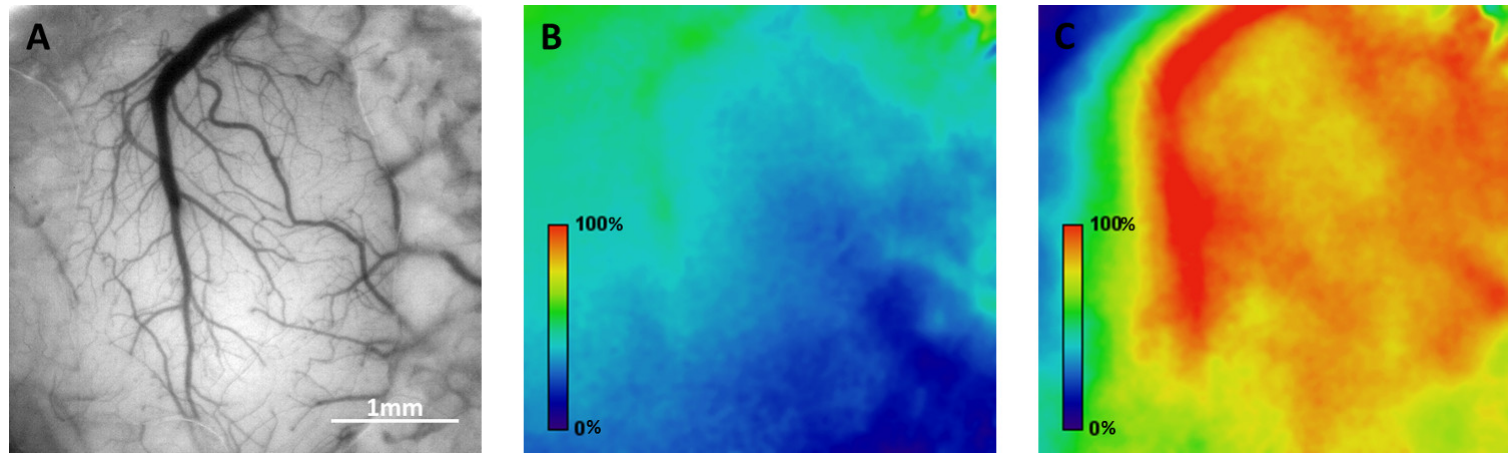

Figure 4. Example of mapping the spatial distribution of functional responses in the cortex of an awake mouse by means of imaging the intrinsic optical signals. A) Bright-field view of the superficial blood vessels through the cranial window. B) Magnitude map of the baseline activity in mobile homecage during a 6-min episode. C) Magnitude map of neuronal activity propagating along somatosensory cortex in response to the vibrissa stimulation at a frequency of $0.05 \mathrm{~Hz}$. Please click here to view a larger version of this figure. 
A

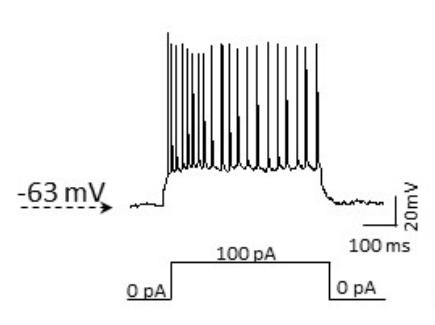

B

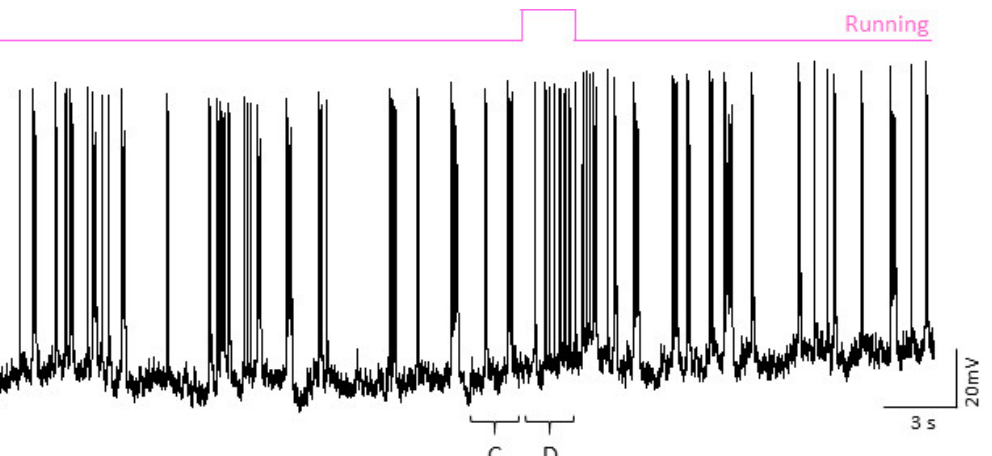

C

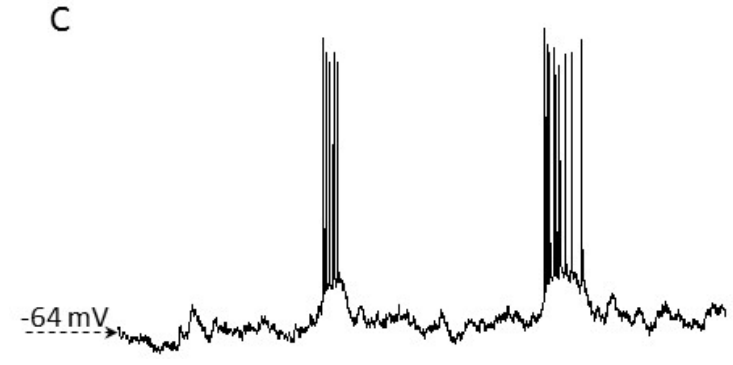

D

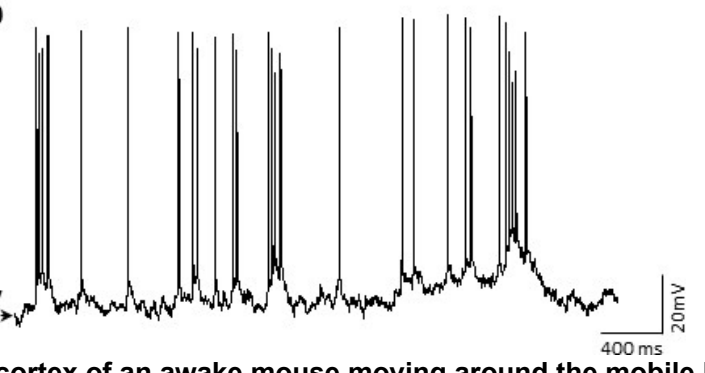

Figure 5. Example of whole-cell patch-clamp recording in the cortex of an awake mouse moving around the mobile homecage. A) Current-clamp recording from a neuron in the mouse cortical layer 2/3. A $0.5 \mathrm{sec}, 100-\mathrm{pA}$ current injection (indicated below the trace) results in a burst of action potentials. The cell showed spike frequency adaptation characteristic for pyramidal neurons. B) Continuous current-clamp recording from the same neuron correlated with the mouse' locomotor activity (shown in pink above the trace). Representative spontaneous activity of the layer $2 / 3$ neuron during periods of the mouse's resting (C) and running (D). Please click here to view a larger version of this figure.

A

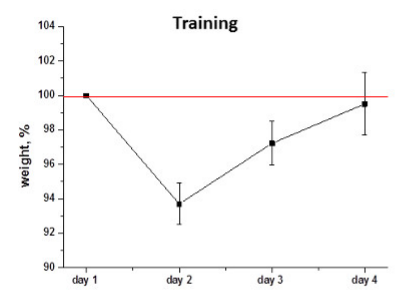

B

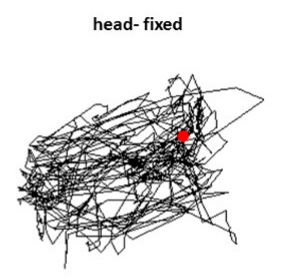

C

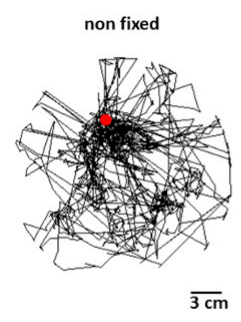

D

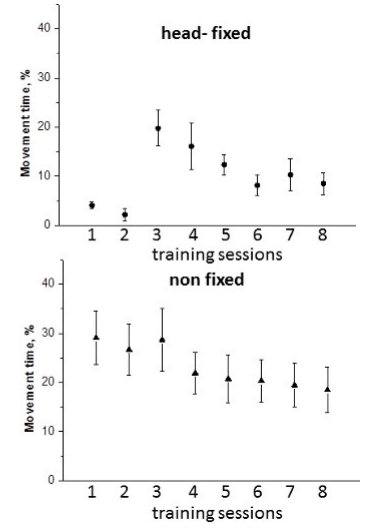

Figure 6. Animal weight loss and locomotor activity of head-fixed/non-fixed mice during training sessions in the mobile homecage. A) Animal weight (mean+SD,\%) before training sessions. Note that weight loss is fully reversed by the $7-8^{\text {th }}$ training session. B) Trajectory of the mouse's horizontal locomotion relative to the mobile homecage, which was extrapolated from the tracked movement of the mobile homecage during $8^{\text {th }}$ training session. C) Tracked movements of a non-head-fixed mouse exploring the round cage during $8^{\text {th }}$ training session. D) Duration of head-fixed (circle) and non-fixed (triangle) mice movement during $1-4^{\text {th }}$ day of training (mean+SD,\%). Note that, on day 4 , head-fixed mice display neither freezing (as on day 1) nor excessive locomotor activity. Please click here to view a larger version of this figure.

\section{Discussion}

To better understand brain physiology and pathology, research must be performed on a variety of preparation complexity levels, utilizing the most appropriate techniques for each preparation. At present, a wide range of neuroscience methodologies (from full-body fMRI to sub-organelle STED microscopy) are readily applied to anaesthetized animals, while experiments on awake and behaving animals have represented a significant methodological challenge.

Here, a novel approach is described where a laboratory animal, despite being firmly head-fixed, can move around an air-lifted mobile homecage and explore its tangible environment under stress-free conditions. The head-fixed behaving animal preparation presented here provides a number of crucial advantages. First, electrophysiological or imaging data obtained with this method are uncompromised neither by anesthesia nor by constrain-induced stress. Positioning of the mouse into the mobile homecage is quick and does not require anesthetizing the animal even transiently. Second, the air-lifted homecage ensures the mechanical stability that is needed to quantify changes in fine neuronal morphology and to record single-cell electrophysiological activity in awake animals. Finally, the mobile homecage's design is more compact in comparison to the 
spherical treadmill, thus allowing positioning the mobile homecage under a standard upright microscope for two-photon imaging or patch-clamp recording in awake mouse's brain.

Firm head fixation in the mobile homecage requires implantation of a specially designed four-winged metal holder, with a round opening in the center for optical or electrical access to the underlying brain region. These metal holders are attached to the skull by means of a combination of glue, dental cement and a small bolt screwed into the skull bone. This surgical procedure was developed based on a large number of previously published procedures, and was found to result in a stable and reproducible cranial window preparation. For in vivo electrophysiological experiments, a moon-shaped window ${ }^{34}$, a small size craniotomy (less than $\left.0.5 \mathrm{~mm}\right)^{32}$, and a drilled glass-covered preparation ${ }^{35}$ have been utilized. Here, the "inverted" cranial window was implanted with either a large ( $3.5 \mathrm{~mm}$ diameter) or small (less than $0.5 \mathrm{~mm}$ diameter) craniotomy. Minimizing brain movement is critical for stable single cell recordings, which is why it is advisable to perform small size craniotomies for electrophysiological experiments. Upon implantation of the cranial window for optical imaging experiments, the animals are allowed to recover for at least 2 or 3 weeks, during which period the window first transiently loses its transparency and then regains it (with a 50-70\% yield, depending on the genetic background of the mouse strain). Transparency of the cranial window and stability of the dental cement "cap" attached to the skull can be verified by means of a regular binocular microscope and physical inspection during animal handling. At the end of the 2-3 week recovery period, those animals that exhibit any signs of residual post-operational inflammation or mechanical defects in the dental cement should be excluded from the experiments and terminated.

The optimal age for starting training the mice is 2-4 months (corresponding to the body weight of $20-40 \mathrm{~g}$ ). In younger animals, anchoring of the dental cement "cap" to the skull can be unreliable, which may decrease its resilience to the mechanical stress that is imposed by locomotion of the head-fixed mouse in the mobile homecage. Although male and female mice appear equally willing to navigate in mobile homecage, there is a tendency to achieve better percentage of cranial windows regaining their transparency in female mice (data not shown). Hence, in order to ensure a balanced mix of genders in the cohort of animals selected for imaging, implanting cranial windows in approximately $30 \%$ more male mice is recommended. Social interactions are known to improve the animals' well-being and reduce stress, therefore it is advisable that littermates are operated and trained in parallel and kept together in group-housing cages.

In contrast to the procedures published for the spherical treadmill preparation ${ }^{13}$, the method utilizing the mobile homecage does not require anesthetizing the mouse at the moment of head fixation. This difference is important because it allows to rule out any residual effects that even a brief and "light" anesthesia episode is likely to have on the physiological measurements obtained shortly after. Indeed, even though in the studies where head fixation was done under anesthesia and the actual experiments were started after a brief waiting period ${ }^{13}$, one cannot exclude possible long-lasting effects of the brief anesthesia episode on the experimental data. Other studies have relied on water deprivation for systematic habituation of the animals to head fixation and used water reward as the means of motivating the animal to remain immobile ${ }^{36}$. However, the reward-based head fixation method limits the choice of applicable behavioral tests and, importantly, occupies one of the wellestablished stimulus-reward associations. In contrast, the method of mouse habituation to head fixation in mobile homecage does not require water deprivation and subsequent reward.

Supplementing the mobile homecage with a water delivery system is recommended for long-lasting experiments. The animal training sessions and experiments presented here were done during daytime (between 8 a.m. and 6 p.m.), which corresponds to the physiologically passive period for those mice that are kept under the standard 12-hr light schedule (lights on at 6 a.m. and off at 6 p.m.). Since the water intake is directly associated with the mouse's activity, during the passive period mice do not require water delivery if the duration of a training/imaging/recording session does not exceed $2 \mathrm{hr}$. In addition to the timing and duration of the training sessions, one needs to address the issue of the optimal number of sessions required for habituating the animals to mobile homecage. To this end, two criteria were used to evaluate stress induced by head fixation procedures: i) weight loss, and ii) locomotor activity level. As shown in Figure 6, weight loss reaches the average level of $6 \%$ on training day 2 , and is completely reversed by training day 4 (Figure 6A). Consistently with the weigh dynamics, the locomotor activity level of head-fixed animals is suppressed on the first day of training but stabilizes by training day 4 (Figure 6D). Based on these measurements, we suggest that the minimal duration of the mouse training period on mobile homecage is 4 days, as described in the protocol hereby.

Use of the air-lifted, flat-floored mobile homecage allows adding complex tasks (sensorimotor, perceptional, and cognitive) to the training paradigms for head-fixed mice. In the present study two protocols of behavioral tests are presented. Both protocols utilize odor cues and can be combined with longitudinal imaging/recordings in the mouse cortex. Although the mobile homecage is manufactured from nonabsorbent materials, one still needs to take into account possible interferences between the smell of the device and test odor(s). Another factor that may interfere with visual/tactile cues of a behavioral experiment is the junction between the wall and the insert, which is not seamless and may, therefore, be perceived by the animal as a landmark. It is worth noticing here that, in order to minimize animal's distress during such interventions as placement of an odor-presenting cotton to the mobile homecage wall, the experimentalist should practice to perform such interventions as quickly as possible and avoid prolonged handling of the carbon cage. Alternative strategies for novel smell/object presentation are conceivable, e.g., placing hydrogel-based solution drops or objects (such as food chips) onto small shelves attached to the inner surface of the carbon cage wall at the height compatible with the animal's head positioning.

Mobile homecage allows head-fixed animals to perform a wide range of two-dimensional movements including horizontal locomotion, situp, grooming, whisking, licking, nose-poking, skilled front paw movements, and wall touching with forelimbs, as illustrated in the present study. Using mobile homecage and the protocols presented here, researchers can study the sensorimotor neuronal system with a high level of control over both the stimulation conditions and the behavioral read-outs. Furthermore, studies of cognitive abilities in awake mice can be performed during conditioning, spatial navigation and decision-making tasks.

There are several practical limitations of this method. First, a significant amount of pressurized air is needed to achieve the homecage-lifting power and to perform long-lasting experiments. Second, the mobile homecage in its present implementation is only $18 \mathrm{~cm}$ in diameter, and therefore provides a relatively small and simple space in comparison to virtual reality, where a complex experimental environment can be designed without any spatial restrictions. Third, during whisker stimulation and reward-based experiments presented here, a device was used that limits the possibility the wall-contact for the mouse. Addition of an external visual or sensory stimulation channel (such as an eye-directed light projector) would require designing a more ergonomic and compact device in comparison to the multiple-screen or dome-projection solutions that have been used in the spherical treadmill experiments. 
In summary, the use of the head-fixed mice moving in the air-lifted mobile homecage greatly facilitates the studies that combine cellular, molecular and behavioral levels of observation and manipulation within a single experiment. Specific applications illustrated here include twophoton microscopic imaging, intrinsic optical signal imaging and patch-clamp recordings in non-anesthetized behaving mice. It is expected that this approach will open new horizons in experimentation on awake, behaving mouse and serve as a useful tool for both drug development and basic research of brain function.

\section{Disclosures}

The authors have nothing to disclose.

\section{Acknowledgements}

The authors thank Prof. Eero Castren for his valuable comments on the manuscript. The work is supported by grants from The Academy of

Finland, Centre for International Mobility of Finland, and Finnish Graduate School of Neuroscience (Brain and Mind Doctoral Program).

\section{References}

1. Li, N. et al. mTOR-dependent synapse formation underlies the rapid antidepressant effects of NMDA antagonists. Science. 329, 959-964, doi:10.1126/science.1190287 (2010).

2. Sitdikova, G. et al. Isoflurane suppresses early cortical activity. Annals of Clinical and Translational Neurology. 1 (1), 15-26, doi:10.1002/ acn3.16 (2013).

3. Helmchen, F., Fee, M. S., Tank, D. W., \& Denk, W. A miniature head-mounted two-photon microscope. high-resolution brain imaging in freely moving animals. Neuron. 31, 903-912, doi:10.1016/S0896-6273(01)00421-4 (2001).

4. Piyawattanametha, W. et al. In vivo brain imaging using a portable $2.9 \mathrm{~g}$ two-photon microscope based on a microelectromechanical systems scanning mirror. Optics letters. 34, 2309-2311, doi:10.1364/OL.34.002309 (2009).

5. Sawinski, J., Wallace, D. J., Greenberg, D. S., Grossmann, S., Denk, W., \& Kerr, J. N. D. Visually evoked activity in cortical cells imaged in freely moving animals. Proceedings of the National Academy of Sciences of the United States of America. 106, 19557-19562, doi:10.1073/ pnas.0903680106 (2009)

6. Fee, M. S. Active stabilization of electrodes for intracellular recording in awake behaving animals. Neuron. 27, 461-468, doi:10.1016/ S0896-6273(00)00057-X (2000).

7. Greenberg, D., Houweling, A., \& Kerr, J. Population imaging of ongoing neuronal activity in the visual cortex of awake rats. Nat Neurosci. 11 (7), 749-751, doi:10.1038/nn.2140 (2008).

8. Fujiwara-Tsukamoto, Y. et al. Reinforcing operandum: rapid and reliable learning of skilled forelimb movements by head-fixed rodents. Journal of Neurophysiology. 108, 1781-1792, doi:10.1152/jn.00356.2012 (2012).

9. Scott, B. B., Brody, C. D., \& Tank, D. W. Cellular Resolution Functional Imaging in Behaving Rats Using Voluntary Head Restraint. Neuron 80, 371-384, doi:10.1016/j.neuron.2013.08.002 (2013).

10. Dombeck, D. A., Harvey, C. D., Tian, L., Looger, L. L., \& Tank, D. W. Functional imaging of hippocampal place cells at cellular resolution during virtual navigation. Nat Neurosci. 13, 1433-1440, doi:10.1038/nn.2648 (2010).

11. Parry, T. J., \& McElligott, J. G. A method for restraining awake rats using head immobilization. Physiolog., \& behavior. 53 (5), 1011-5 (1993).

12. Brecht, M., Schneider, M., Sakmann, B., \& Margrie, T. W. Whisker movements evoked by stimulation of single pyramidal cells in rat motor cortex. Nature. 427 (6976), 704-10, doi:10.1038/nature02266 (2004).

13. Van Looij, M. A. J., Liem, S.-S., van der Burg, H., van der Wees, J., De Zeeuw, C. I., \& van Zanten, B. G. A. Impact of conventional anesthesia on auditory brainstem responses in mice. Hearing research. 193, 75-82, doi:10.1016/j.heares.2004.02.009 (2004).

14. Hentschke, H., Schwarz, C., \& Antkowiak, B. Neocortex is the major target of sedative concentrations of volatile anaesthetics: strong depression of firing rates and increase of GABAA receptor-mediated inhibition. The European journal of neuroscience. 21, 93-102, doi:10.1111/j.1460-9568.2004.03843.x (2005).

15. Margrie, T. W., Brecht, M., \& Sakmann, B. In vivo, low-resistance, whole-cell recordings from neurons in the anaesthetized and awake mammalian brain. Pflugers Archiv : European journal of physiology. 444, 491-498, doi:10.1007/s00424-002-0831-z (2002).

16. Crochet, S., \& Petersen, C. C. H. Correlating whisker behavior with membrane potential in barrel cortex of awake mice. Nat Neurosci. 9, 608610, doi:10.1038/nn1690 (2006)

17. Houweling, A. R., \& Brecht, M. Behavioural report of single neuron stimulation in somatosensory cortex. Nature. 451, 65-68, doi:10.1038/ nature06447 (2008).

18. Poulet, J. F. A., \& Petersen, C. C. H. Internal brain state regulates membrane potential synchrony in barrel cortex of behaving mice. Nature. 454, 881-885, doi:10.1038/nature07150 (2008).

19. Bryant, J. L., Roy, S., \& Heck, D. H. A technique for stereotaxic recordings of neuronal activity in awake, head-restrained mice. Journal of neuroscience methods. 178, 75-79, doi:10.1016/j.jneumeth.2008.11.014 (2009).

20. De Kock, C. P. J., \& Sakmann, B. Spiking in primary somatosensory cortex during natural whisking in awake head-restrained rats is cell-type specific. Proceedings of the National Academy of Sciences of the United States of America. 106, 16446-16450, doi:10.1073/ pnas.0904143106 (2009)

21. Dombeck, D. A., Khabbaz, A. N., Collman, F., Adelman, T. L., \& Tank, D. W. Imaging large-scale neural activity with cellular resolution in awake, mobile mice. Neuron. 56, 43-57, doi:10.1016/j.neuron.2007.08.003 (2007).

22. Hentschke, H., Haiss, F., \& Schwarz, C. Central signals rapidly switch tactile processing in rat barrel cortex during whisker movements Cerebral cortex. 16, 1142-1156, doi:10.1093/cercor/bhj056 (2006).

23. Stüttgen, M. C., Rüter, J., \& Schwarz, C. Two psychophysical channels of whisker deflection in rats align with two neuronal classes of primary afferents. J. neuroscience. 26, 7933-7941, doi:10.1523/JNEUROSCI.1864-06.2006 (2006). 
24. Connor, D. H., Peron, S. P., Huber, D., \& Svoboda, K. Neural activity in barrel cortex underlying vibrissa-based object localization in mice. Neuron. 67, 1048-1061, doi:10.1016/j.neuron.2010.08.026 (2010).

25. Drew, P. J., Shih, A. Y., \& Kleinfeld, D. Fluctuating and sensory-induced vasodynamics in rodent cortex extend arteriole capacity. Proceedings of the National Academy of Sciences of the United States of America. 108 (20), 8473-8478, doi:10.1073/pnas.1100428108 (2011).

26. Harvey, C. D., Collman, F., Dombeck, D. A., \& Tank, D. W. Intracellular dynamics of hippocampal place cells during virtual navigation. Nature. 461, 941-946, doi:10.1038/nature08499 (2009).

27. Chen, G., King, J. A., Burgess, N., \& O'Keefe, J. How vision and movement combine in the hippocampal place code. Proceedings of the National Academy of Sciences of the United States of America. 110 (1), 378-83, doi:10.1073/pnas.1215834110 (2013).

28. Harvey, C. D., Coen, P., \& Tank, D. W. Choice-specific sequences in parietal cortex during a virtual-navigation decision task. Nature. 484 (7392), 62-68, doi:10.1038/nature10918 (2012).

29. Holtmaat, A. et al. Long-term , high-resolution imaging in the mouse neocortex through a chronic cranial window. Nat Protoc. 4 (8), 19-22, doi:10.1038/nprot.2009.89 (2009).

30. Mostany, R., \& Portera-Cailliau, C. A craniotomy surgery procedure for chronic brain imaging. J. Vis. Exp. (12), doi:10.3791/680 (2008).

31. Portera-Cailliau, C., Trachtenberg, J. T., de Paola, V., Svoboda, K., Wilbrecht, L., \& Holtmaat, A. Imaging Neocortical Neurons through a Chronic Cranial Window. Cold Spring Harbor Protocols. 2012, pdb.prot069617-pdb.prot069617, doi:10.1101/pdb.prot069617 (2012).

32. Garaschuk, O., Milos, R.-I., \& Konnerth, A. Targeted bulk-loading of fluorescent indicators for two-photon brain imaging in vivo. Nat Protoc. 1 (1), 380-6, doi:10.1038/nprot.2006.58 (2006).

33. Barry, P. H. JPCalc, a software package for calculating liquid junction potential corrections in patch-clamp, intracellular, epithelial and bilayer measurements and for correcting junction potential measurements. Journal of neuroscience methods. 51 (1), 107-16 (1994).

34. Golshani, P., Gonçalves, J. T., Khoshkhoo, S., Mostany, R., Smirnakis, S., \& Portera-Cailliau, C. Internally mediated developmental desynchronization of neocortical network activity. The Journal of neuroscience. 29 (35), 10890-9, doi:10.1523/JNEUROSCI.2012-09.2009 (2009).

35. Polack, P.-O., Friedman, J., \& Golshani, P. Cellular mechanisms of brain state-dependent gain modulation in visual cortex. Nat Neurosci. 16 (9), 1331-9, doi:10.1038/nn.3464 (2013).

36. Schwarz, C. et al. The head-fixed behaving rat--procedures and pitfalls. Somatosensor., \& motor research. 27, 131-148, doi:10.3109/08990220.2010.513111 (2010). 